3 or more PIDs. $70.9 \%$ were psychotropic drugs. $53.7 \%$ of them were initiated by doctors working in our hospital, $86.4 \%$ of which by a senior doctor versus $13.6 \%$ by a resident.

Conclusions This study shows that a significant proportion of PIDs are initiated in our hospital. To improve practise, pharmacists have to make doctors aware of PIDs and suggest therapeutic alternatives before treatment is started. If PIDs are prescribed, pharmacists should formulate pharmaceutical interventions.

We will add this criterion to our trigger tool which selects highrisk prescriptions.

No conflict of interest.

\section{GRP-099 INCIDENCE OF DRUG INTERACTIONS IN A CARDIOLOGY DEPARTMENT}

doi:10.1136/ejhpharm-2013-000276.099

A Tóbel, I Higyisán. Bajcsy-Zsilinszky Hospital, Institute Pharmacy, Budapest, Hungary

Background Starting in 2007, the Pharmacy Institute at BajcsyZsilinszky Hospital in Budapest was the first healthcare institution in Hungary to use centralised medicines Daily Dose System (DDS).

The number of medicines administered to a patient may increase the probability of drug interactions. If physicians prescribe treatment without due foresight this may cause subsequent problems for the patient.

Purpose Pharmacists are the last cheque-point in the medicines system. The study sought to justify the importance of this by monitoring interactions.

Materials and Methods The incidence of theoretical and clinically relevant interactions was followed on the cardiology department at Bajcsy-Zsilinszky Hospital in a four-week period cross-sectional study. During this period, the drug treatment and the potential interactions were examined by using NovoHosp.win software.

Results A total of 218 patients were registered in the study, gender distribution of the sample: 100 women (46\%) and 118 men (54\%). A total of 1,893 drugs were prescribed, an average of 9 drugs per patient. The NovoHosp.win software found 603 interactions, which was an average of 3 interactions per patient. 174 patients had at least one possible interaction, but clinically relevant problems (increased APTT and INR values, potassium level differences and uric acid changes) had only arisen in 25 patients, 8 women (32\%) and 17 men (68\%). The software indicated 4 theoretical and 1 clinically relevant interactions in this patient group. The relevant interactions were classified as follows: potassium level differences 19\%, uric acid changes $22 \%$, APTT abnormalities $37 \%$, changes in INR $22 \%$.

Conclusions In the present study, 25 patients had 30 relevant interactions, as a result of which medicines were changed on 22 occasions. Changes in the dose, dose adjustments or drug substitution abolished the interactions. The study also demonstrates the importance of cooperation between hospital/clinical pharmacists and physicians.

No conflict of interest.

\section{GRP-100 INCIDENCE OF ERRORS IN DRUG DOSAGE ACCORDING TO KIDNEY FUNCTION-ESTIMATING EQUATIONS IN MEDICAL INPATIENTS}

doi:10.1136/ejhpharm-2013-000276.100

'L Rojas, ${ }^{2} \mathrm{~N}$ Severino, ${ }^{2 \mathrm{R}}$ Mellado. ${ }^{1}$ Faculty of Medicine. Pontificia Universidad Católica de Chile, Internal Medicine, Santiago, Chile; ${ }^{2}$ Faculty of Pharmacy. Pontificia Universidad Católica de Chile, Pharmacy, Santiago, Chile

Background Inpatients frequently require dose adjustments of medicines due to acute changes in renal function. The FDA recommend adjusting medicines according to the estimated glomerular filtration obtained with the Cockcroft-Gault formula. However the Modification of Diet in Renal Disease (MDRD) study equation is widely recognised as more accurate than Cockcroft-Gault, which confuses clinicians because they do not know its utility for adjusting drug doses.

Purpose To compare the incidence in inpatients of medicine dosing errors depending on the type of equation used to estimate it: Cockcroft-Gault or MDRD.

Materials and Methods A cross-sectional study was conducted in a low complexity unit. Patients were included with impaired renal function who were not on haemodialysis.

We used the FDA guidelines to determine the incidence of errors.

Fisher's test was used to compare the groups, with statistical significance level $<0.05$.

Results We included 56 inpatients and 214 prescriptions. 58\% were women and $68 \%$ were older than 65 . We detected $42 \%$ and $28 \%$ of errors using CG and MDRD, respectively ( $p=0.014)$. The most common error was an overdose $(79 \%)$ followed by an underdose $(12 \%)$ and contraindication $(9 \%)$.

Further analysis found that the difference between the two equations occurred only in the following subgroups of patients: patients with mild to moderate impairment of renal function $38 \%$ versus $23 \%, p=0.03)$, older than 65 years $(51 \%$ versus $30 \%$, $p=0.01)$ and low body weight ( $37 \%$ versus $31 \%, p=0.04)$. The distribution of types of errors was similar in the three subgroups.

Conclusions The percentage of dosing error for both methods was similar to that reported in the literature.

The two equations were not discordant except in the elderly, in patients with low body weight and with mild renal dysfunction. This could explain why there were differences in the incidence of medicine errors in these subgroups.

In the absence of a gold standard to assess the acute deterioration of renal function and considering the limitations in estimating renal function with these equations, clinicians should include clinical judgement when determining the dose for each patient. The dose should be determined by weighing the risk of toxicity with higher doses versus the risk of treatment failure with lower doses, especially in elderly and low body weight patients.

No conflict of interest.

\section{GRP-101 INSULIN: IMPROVING PRESCRIBING SAFETY}

doi:10.1136/ejhpharm-2013-000276.101

DN Wigg, V Ruszala. North Bristol NHS Trust, Pharmacy Department, Bristol, UK

Background Insulin has been defined as one of the highest risk medicines worldwide, [1] with a 2009 national UK audit demonstrating prescribing errors in $19.5 \%$ of in-patient insulin prescriptions. [2] The NPSA (National Patient Safety Agency) Rapid Response Report, issued in June 2010, further highlighted errors in the administration of insulin by clinical staff and called for immediate action to improve insulin prescribing. [2]

Purpose In 2010, an audit of insulin prescribing was conducted at North Bristol NHS Trust (NBT), using the Patient Safety First 'insulin prescription bundle' data collection tool that focused on five key safety-critical prescribing elements. [4] Following the results of the 2010 audit and NPSA alert, an insulin prescription chart was developed with the aim of significantly improving insulin prescribing. Materials and Methods On 4th October 2012, the impact of the NBT insulin prescription chart was examined during a one-day cross-sectional audit (incorporating all specialities), using a special data collection form developed from the insulin prescription bundle'. [4] This incorporated five key audit standards:

a. All prescriptions written by brand name with the word 'insulin' included

b. The word 'Units' written in full 